\title{
Introduction to research topic - Brain connectivity analysis: investigating brain disorders. Part 1: the review articles
}

\author{
Barry Horwitz ${ }^{*}$ and Silvina G. Horovitz ${ }^{2}$ \\ Brain Imaging and Modeling Section, National Institute on Deafness and Other Communication Disorders, National Institutes of Health, Bethesda, MD, USA \\ Human Motor Control Section, National Institute of Neurological Disorders and Stroke, National Institutes of Health, Bethesda, MD, USA \\ *Correspondence: horwitzb@nidcd.nih.gov
}

In the past few years, advances in human structural and functional neuroimaging, especially with respect to magnetic resonance imaging, have resulted in an explosion of studies exploring the anatomical and functional connectivities between different regions of the brain. More and more studies have employed resting and task-related connectivity analyses to assess functional interactions, and other studies have used diffusion-weighted tractography to examine the organization and integrity of white matter tracts. Many of these studies have addressed normal human function, but recently, a number of investigators have turned their attention to examining brain disorders. We are on the verge of being able to better understand the pathophysiology of neurological and psychiatric disorders and the effect of treatments on brain function. The study of brain disorders is a complex endeavor: not only does it require understanding the normal brain, and the regions involved in a particular function, but also it needs a deeper understanding of brain networks and their dynamics. Moreover, in many cases, disorders are progressive, and thus imaging could potentially become a source of biomarkers for early detection and assessment of the course of a disease (Horwitz and Rowe, 2011), and for evaluating potential treatments. The articles that appear in this special topics ebook represent the current status of the applications that employ brain connectivity analysis to investigate brain disorders.

Although certain types of connectivity analyses have become very popular recently [e.g., resting state fMRI functional connectivity - see the special topics issue of Frontiers in Systems Neuroscience edited by Uddin and Menon (2010)], we have chosen to cover articles that represent the broadest possible set of connectivity methods, employing the widest possible spectrum of imaging techniques, and applied to the full range of neurological, psychiatric, and developmental disorders. In this way, we hope that readers acquire an understanding of how these relatively new connectivity analysis methods enable investigators to address brain disorders from a network perspective. Even pathology localized to one area of the brain can lead to dysfunctional network behavior, since no brain region is an island onto itself. The insights offered by the network paradigm have, therefore, important ramifications for using neuroimaging to help detect and diagnose brain disorders, interpret the symptoms of many disorders, and assess potential treatments.

Two types of articles comprise this special topics ebook - review articles and papers that include primarily the results of original research. Here, we provide a brief overview of the review articles. See the Horovitz and Horwitz editorial (Introduction to research topic - Brain connectivity analysis: investigating brain disorders. Part 2: original research articles) for a corresponding overview of the research papers.
The review articles can be roughly divided into (1) those that focus on a connectivity method, and use one or more disorders to illustrate the method, and (2) those whose emphasis is placed on a particular disorder, and discuss a number of relevant functional and structural connectivity studies investigating the disorder. In the first category of reviews, Rowe (2010) provides an overview of fMRI-based functional and effective connectivity, with a focus on its applications to neurological disorders. One of the most widely used effective connectivity techniques is dynamic causal modeling (DCM; Friston et al., 2003), and Seghier et al. (2010) present an extensive review of its application to all brain disorders. This article also includes a useful introduction to Bayesian model selection (Penny et al., 2004), a key component of DCM. Another "functional" connectivity method that has been applied to investigate brain disorders is transcranial magnetic stimulation (TMS), and the article by Hampson and Hoffman (2010) contains a nice overview of the method, along with a review of its applications to psychiatric and neurological disorders. In TMS, an externally applied changing magnetic field is used to induce electrical stimulation in a cortical brain area which propagates through long-range connections to other brain regions. David et al. (2010) discuss a similar method, direct electrical stimulation (DES), applied using intracranial electrodes in some epileptic patients during presurgical evaluation prior to epileptogenic tissue resection. DES is applied directly to cortical tissue and tracts with extremely well-defined spatial definition, and in this sense, it provides human data that are unattainable by any other method. Epilepsy also provides the basis for two other reviews. Wendling et al. (2010) discuss a variety of functional connectivity techniques that have been applied to electrophysiological data obtained from scalp and intracerebral EEG recordings, whereas the review by Lemieux et al. (2011) offers a broad overview of both EEG-based and fMRI-based functional and effective connectivity analyses. Finally, Alexander-Bloch et al. (2010) provide a useful overview of the basic concepts and mathematics of graph theory as applied to fMRI resting data. As an example of graph theory as applied to resting state fMRI, they present results of a study comparing childhood-onset schizophrenia with normal controls, showing disrupted modularity and local connectivity in the patients.

The second category of review articles focuses on disorders. Schipul et al. (2011) provide a detailed review of autism. In many ways autism represents the classic example of a disorder where analyses of imaging data at the single brain region level yielded very little insight, but network analyses have proven quite fruitful in furthering our understanding of the condition. A second brain disorder that is reviewed here is coma (and related disorders of consciousness). Noirhomme et al. (2010) 
show that such studies can shed important light on what mediates conscious awareness. Two articles address stroke, in particular focusing on using brain connectivity analyses to assess functional recovery. Westlake and Nagarajan (2011) provide an overview of functional connectivity in relation to motor performance. Their review emphasizes PET, fMRI, and EEG/ MEG studies. In the other review, Johansen-Berg et al. (2010) examine white matter connectivity results, obtained using diffusion tensor imaging (DTI). Their paper includes information on how this technique can be used to test for dynamic changes in structural connectivity with learning or with recovery from the effects of a stroke.

\section{ACKNOWLEDGMENTS}

This work was supported by the Intramural Research Programs of NIDCD (BH) and NINDS (SGH).

\section{REFERENCES}

Alexander-Bloch, A. F., Gogtay, N., Meunier, D., Birn, R., Clasen, L., Lalonde, F., Lenroot, R., Giedd, J., and Bullmore, E. T. (2010). Disrupted modularity and local connectivity of brain functional networks in childhood-onset schizophrenia. Front. Syst. Neurosci. 4:147. doi: $10.3389 /$ fnsys. 2010.00147

David, O., Bastin, J., Chabardes, S., Minotti, L., and Kahane, P. (2010). Studying network mechanisms using intracranial stimulation in epileptic patients. Front. Syst. Neurosci. 4:148. doi: $10.3389 /$ fnsys. 2010.00148

Friston, K. J., Harrison, L., and Penny, W. (2003). Dynamic causal modelling. Neuroimage 19, 1273-1302.

Hampson, M., and Hoffman, R. E. (2010). Transcranial magnetic stimulation and connectivity mapping: tools for studying the neural bases of brain disorders. Front. Syst. Neurosci. 4:40. doi: 10.3389/fnsys.2010.00040

Horwitz, B., and Rowe, J. B. (2011). Functional biomarkers for neurodegenerative disorders based on the network paradigm. Prog. Neurobiol. 95, 505-509.
Johansen-Berg, H., Scholz, J., and Stagg, C. J. (2010). Relevance of structural brain connectivity to learning and recovery from stroke. Front. Syst. Neurosci. 4:146. doi: 10.3389/fnsys.2010.00146

Lemieux, L., Daunizeau, J., and Walker, M. C. (2011). Concepts of connectivity and human epileptic activity. Front. Syst. Neurosci. 5:12. doi: 10.3389/fnsys.2011.00012

Noirhomme, Q., Soddu, A., Lehembre, R., Vanhaudenhuyse, A., Boveroux, P., Boly, M., and Laureys, S. (2010). Brain connectivity in pathological and pharmacological coma. Front. Syst. Neurosci. 4:160. doi: 10.3389/fnsys.2010.00160

Penny, W. D., Stephan, K. E., Mechelli, A., and Friston, K. J. (2004). Comparing dynamic causal models. Neuroimage 22, 1157-1172.

Rowe, J. B. (2010). Connectivity analysis is essential to understand neurological disorders. Front. Syst. Neurosci. 4:144. doi: 10.3389/fnsys.2010.00144

Schipul, S. E., Keller, T. A., and Just, M. A. (2011). Inter-regional brain communication and its disturbance in autism. Front. Syst. Neurosci. 5:10. doi: 10.3389/ fnsys. 2011.00010

Seghier, M. L., Zeidman, P., Neufeld, N. H., Leff, A. P., and Price, C. J. (2010). Identifying abnormal connectivity in patients using dynamic causal modeling of FMRI responses. Front. Syst. Neurosci. 4:142. doi: 10.3389/fnsys.2010.00142

Uddin, L. Q., and Menon, V. (2010). Introduction to special topic - resting-state brain activity: implications for systems neuroscience. Front. Syst. Neurosci. 4:37. doi: 10.3389/fnsys.2010.00037

Wendling, F., Chauvel, P., Biraben, A., and Bartolomei, F. (2010). From intracerebral EEG signals to brain connectivity: identification of epileptogenic networks in partial epilepsy. Front. Syst. Neurosci. 4:154. doi: 10.3389/fnsys.2010.00154

Westlake, K. P., and Nagarajan, S. S. (2011). Functional connectivity in relation to motor performance and recovery after stroke. Front. Syst. Neurosci. 5:8. doi: 10.3389/ fnsys.2011.00008

Received: 25 January 2012; accepted: 27 January 2012; published online: 13 February 2012. Citation: Horwitz B and Horovitz SG (2012) Introduction to research topic - Brain connectivity analysis: investigating brain disorders. Part 1: the review articles. Front. Syst. Neurosci. 6:3. doi: 10.3389/fnsys.2012.00003

Copyright (c) 2012 Horwitz and Horovitz. This is an open-access article distributed under the terms of the Creative Commons Attribution Non Commercial License, which permits non-commercial use, distribution, and reproduction in other forums, provided the original authors and source are credited. 